\title{
Optimizing Paced Ventricular Function in Patients with and without Repaired Congenital Heart Disease by Contractility-Guided Lead Implant
}

PETER P. KARPAWICH, M.Sc., M.D., HARINDER SINGH, M.D., and KATHLEEN ZELIN, R.N., M.S.N., C.P.N.P.

From the Section of Cardiology, Department of Pediatrics, The Children's Hospital of Michigan, Wayne State University School of Medicine, Detroit, Michigan

Background: This study evaluates the concept of optimizing ventricular pacing in regard to functional cardiac response. Lead implant based on physiologic variables of contractility at various sites was performed in patients with and without congenital heart disease (CHD). Since right ventricular apical pacing may adversely alter contractility and myocellular function, septal and outflow tract pacing have been advocated. However, there are few studies in the young and essentially none in those with CHD.

Methods: A total of 113 consecutive patients with and without repaired CHD, aged two to 51 (median 16), some with preexisting epicardial pacemakers, underwent transvenous pacemaker implant using standard sensing/pacing indices plus measurements of pressures, QRS, and contractility responses at each of five predefined potential ventricular implant sites: apex, inflow-, low-, mid-, and infundibular/outflow-septal with each patient serving as his/her own control. Implant was at the site of best contractility with activefixation, low-threshold steroid-eluting leads.

Results: Measured contractility indices varied up to 31\% (mean 12\%) between sites per patient. Septal regions (mid-, inflow-, and low-) were associated with the most optimal and right ventricular epicardial showed the worst contractility $(P<0.05)$ responses. Apex was optimal in some CHD patients. Threshold and sensing were comparable up to 11 years (mean 7) postimplant regardless of septal site.

Conclusion: There is no single "sweet spot" for optimal ventricular pacing, and the best implant sites are patient and CHD variable. Current lead designs ensure chronic stability/performance regardless of site. Proactive contractility-guided pacing implant can optimize chronic paced ventricular function. (PACE 2015; 38:54-62)

pediatrics, pacing, hemodynamics

\section{Introduction}

The original applications of transvenous ventricular pacing were performed with endocardial leads having limited electrode fixation capabilities which resulted in frequent dislodgements. In this regard, the right ventricular apex offered technical simplicity of implant with stability of electrode performance, with ready-application to pacing children and young adults. ${ }^{1-4}$ However, initiation of paced electrical impulse propagation from this apical region is associated more with muscle than His-Purkinje conduction, causing asynchronous right and left ventricular (LV) contraction. Ventricular function may be modified by

\section{Conflict of Interest: none.}

Address for reprints: Peter P. Karpawich, M.Sc., M.D., Cardiac Electrophysiology, The Children's Hospital of Michigan, 3901 Beaubien Blvd, Detroit, MI 48201; e-mail: pkarpawi@dmc.org

Received February 11, 2014; revised August 8, 2014; accepted September 2, 2014.

doi: 10.1111/pace.12521 pacing rate, atrioventricular activation coupling intervals, and pacing site. Therefore, each of these variables has found interest in both animal and clinical studies to improve paced myocardial performance. Right ventricular (RV) apical pacing, with its resultant paced left bundle branch QRS configuration, causes multiple recognized adverse effects that include paradoxical septal motion, elevated LV pressures, decreased contractility, altered coronary flow, decreased cardiac output, and structural myocellular remodeling. ${ }^{5-8}$ Over time, this remodeling may adversely contribute to histopathologic changes that eventually affect function. ${ }^{9-12}$ As advocated as early as 1925, pacing electrode placement in close proximity to the normal conduction system offers the potential for improved ventricular function by optimizing biventricular (BiV) contractility. ${ }^{13}$ More recently, studies have demonstrated that pacing site, and therefore the initiation of ventricular contractility, may be the prime contributor to function. ${ }^{14-16}$ Contractility measurements have included pressurevolume loops as well as the first derivative of 
systolic pressure ${ }^{17-20}$ which additionally has had pertinent impact with interest in resynchronization pacing for heart failure.

With the introduction of improved low threshold and steerable pacing lead designs permitting fixation at nearly any ventricular location, there has been an increased interest in alternate or select site pacing. Various right ventricular sites ranging from the inflow to outflow/infundibular septum, bundle of His, and midseptal moderator band have been studied. ${ }^{21-26}$ Unfortunately, precise identification of each site has not been uniform in the literature, leading to some confusion as to the exact location advocated per study. As a result, "septal” pacing, as a distinct anatomical location, has limited application. The purpose of this study was to evaluate comparative indices of paced systemic ventricular contractility, using the first derivative of systolic pressure $(\mathrm{dP} / \mathrm{dt})$, as well as more standard evaluations of pressures and QRS morphology, among five distinct ventricular lead insertion "septal" as well as apical sites in younger patients with and without repaired congenital heart disease (CHD) undergoing pacemaker implant in order to ascertain the most optimal implant site yielding the best physiologic paced response. Chronic lead performances at these sites were then compared over time.

\section{Methods}

This was a retrospective study conducted at The Children's Hospital of Michigan, Section of Cardiology. The study was approved by the Institutional Review Board, Wayne State University School of Medicine/Detroit Medical Center. The inclusion criteria were all patients who underwent a transvenous pacing system for symptomatic bradycardia associated with atrioventricular block (AVB). Patients included those with repaired $\mathrm{CHD}$ as well as preexisiting right ventricular free wall epicardial pacing systems that were changed to a transvenous system for various indications, including epicardial lead fracture. All patients were studied using the same institutional pacemaker implant protocol, which, especially since many patients had repaired congenital heart, included a hemodynamic catheterization study with venograms to assess vascular access patency.

After informed consent was obtained, all patients received appropriate sedation, heparinized intravenous fluids (2000 units/500 cc), and antibiotic coverage as recommended. Per institutional protocol for device implantation, all patients were intubated with controlled ventilation. This limited any respiratory effects on measured hemodynamic variables. The femoral vein and artery were en- tered using standard percutaneous techniques and catheters inserted to permit continuous systemic arterial and ventricular pressure monitoring as well as to provide temporary pacing as required. Both right and left cardiac hemodynamics were recorded. A flexible quadripolar pacing catheter, EPT SterroCath model 2001 (Boston Scientific, Natick, MA, USA), was placed in the systemic venous ventricle for temporary pacing. The systemic ventricular pressure recording catheter was connected to either a Cathcor Model 2.2, or Sensis system (Siemens, Munich, Germany) with online program capability of measuring instantaneous waveforms of ventricular pressure as well as waveform analysis of indices of ventricular contractility $(\mathrm{dP} / \mathrm{dt}$ and $\mathrm{dP} / \mathrm{dt} / \mathrm{p})$. Continuous surface electrocardiogram (ECG) tracings were recorded.

The initial superior vena cava/innominate venogram performed to delineate vascular patency was stored for viewing. In the anterior-posterior (AP) image, a perpendicular line was then created to intersect the midtricuspid valve with the ventricular septal surface (Fig. 1). This intersect region was defined as the midseptal area approximating the moderator band location in patients with normal right ventricular morphology and comparable ventricular regions in those with congenital corrected transposition of the great arteries (L-TGA) with LV morphology of the systemic venous ventricle. In patients who had undergone the Mustard procedure for D-transposition of the great arteries (D-TGA), the venogram permitted delineation of the LV septal surface to serve as a comparable marker for lead implant. The apical to semilunar valve distance along the septum was then subdivided into the five mentioned regions (Fig. 1). Once the venous hemodynamics and venogram studies were performed, a standard pigtail 4-Fr or 5-Fr hemodynamic catheter (Cook Medical, Bloomington, IN, USA) was then advanced into the systemic arterial ventricle for pressure and contractility measurements. Due to the anticipated short time to evaluate systemic function, combined with a heparinized saline flush solution, activated clotting time (ACT) levels were not routinely obtained.

In a randomized fashion, the flexible pacing catheter was then advanced to each of the five separate potential septal implant sites as previously described: apex, low septum between the defined midseptum and apex, midseptal, infundibular/outflow septum between the midseptum and semi-lunar valve, and inflow septum approximating the region of the bundle of His/proximal septum. Use of biplane fluoroscopy permitted accurate positioning along the septum with avoidance of the more anterior ventricular 

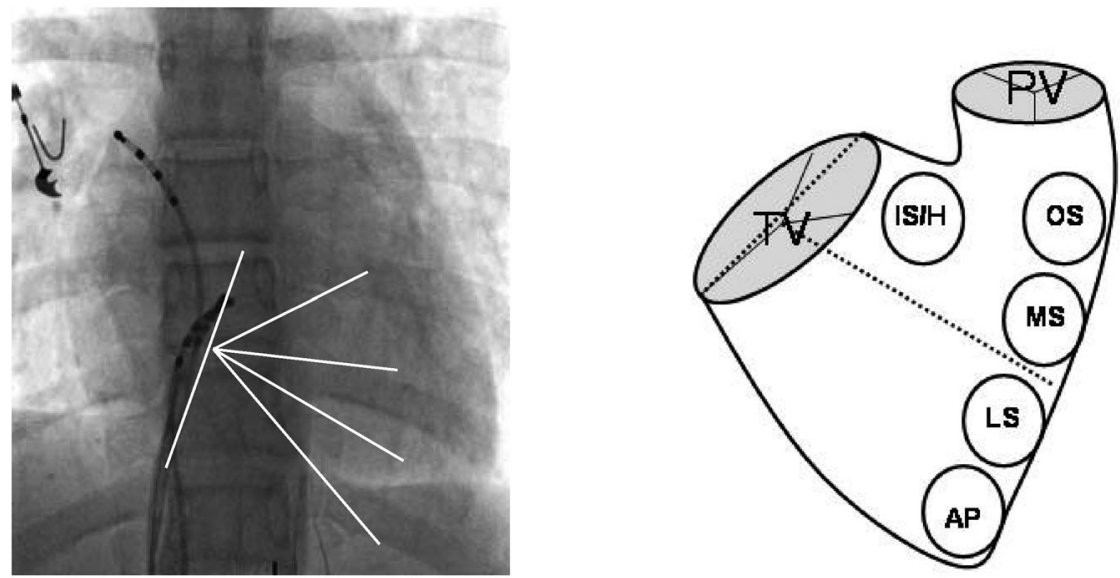

Figure 1. Schematic representation of right ventricular septal lead implant sites as visualized following angiographic appearance in the AP projection (left). Temporary pacing catheters are visualized. Lines are drawn from the midtricuspid valve to intercept the septal surface from apex to outflow to demarcate regions as indicated. $A P=$ apex; $I S / H=$ inflow septum/His; LS $=$ low septum; $M S=$ midseptum; $O S=$ outflow septum; $P V=$ pulmonary valve; $T V=$ tricuspid valve.

free wall. Each patient served as his/her own control. For baseline and for each pacing site, the following protocol was initiated: physiologic indices of ventricular systolic and diastolic pressures, $\mathrm{dP} / \mathrm{dt}, \mathrm{dP} / \mathrm{dt} / \mathrm{p}$, and surface ECG QRS duration and axis were recorded. Among patients with AVB and an adequate intrinsic rate, these baselines values were recorded during the patient's own rhythm. Among those patients with surgical complete AV block and a preexisting epicardial pacing system, baseline values were recorded by programming the pulse generator to slower heart rates only if the intrinsic rate was adequate to prevent any hemodynamic compromise. Otherwise, an ageappropriate paced rate was chosen.

Following 3-5 minutes of pacing with observation to ensure hemodynamic stability, pressures and contractility indices were recorded and the temporary pacing catheter was then randomly positioned at one of the other potential implant sites and comparative physiologic indices recorded during repeat pacing protocols at the same rates. To ensure reliability of the recorded data, at least three separate measurements of contractility variables were obtained per pacing site, and the average recorded. Following permanent lead implant, a repeat contractility measurement was performed. This procedure was repeated at age-appropriate paced rates per patient until data had been obtained from all potential implant sites. Intracardiac electrograms of Purkinje potentials were not specifically sought or evaluated during the mapping protocols. Due to persistent controversy over electrical versus mechanical correlates of contractility, ${ }^{25} \mathrm{dP} / \mathrm{dt}$ was chosen as the single best indicator in those cases when a discrepancy arose between QRS duration and $\mathrm{dP} / \mathrm{dt}$. This was especially true among patients with preexistent surgical bundle branch block.

Following evaluation of best site, a fluoroscopic image was saved, the systemic ventricular catheter was removed, and utilizing standard sterile surgical techniques, a standard subclavicular pocket and pacemaker implant was performed. As determined by vessel size, either the cephalic, subclavian, or axillary vein was entered and the chosen pacing lead inserted and advanced to the appropriate venous ventricle using biplane fluoroscopic guidance. Various steroid-eluting, low threshold, silicone- and polyurethane-insulated, active fixation leads, including the newer M3830 catheter-delivered lead (Medtronic Inc., Minneapolis, MN, USA) were implanted depending on patient size. In each patient, the lead was secured to the preselected area followed by standard sensing and threshold measurements, pulse generator insertion, and pocket closure. Postimplant follow-up ranged from 5 years to 11 years (mean 7).

Statistics compared recorded results at each temporary implant site in each patient and between all patients as a group using the Student paired and nonpaired $t$-test (GraphPad Software, Inc., La Jolla, CA, USA). Significance was described at a $\mathrm{P}$ value $<0.05$. 
A

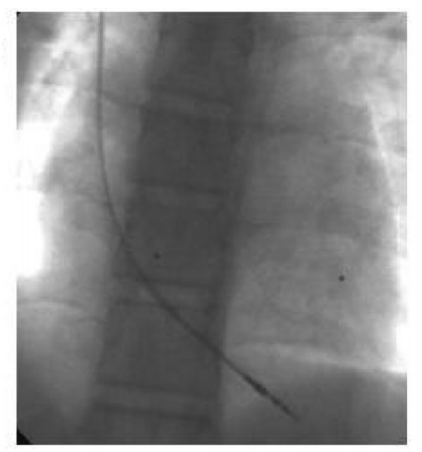

C

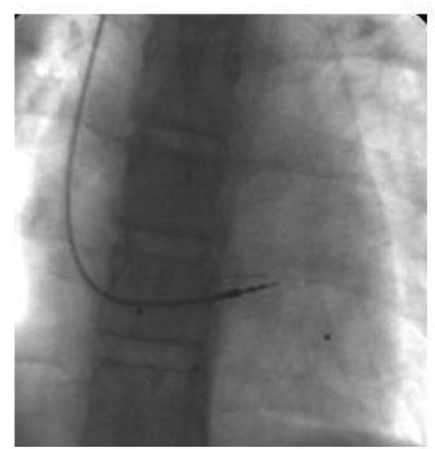

B

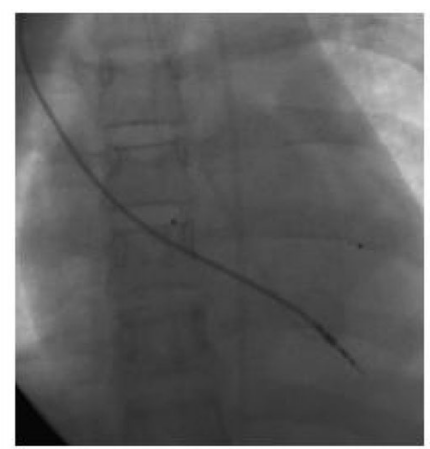

D

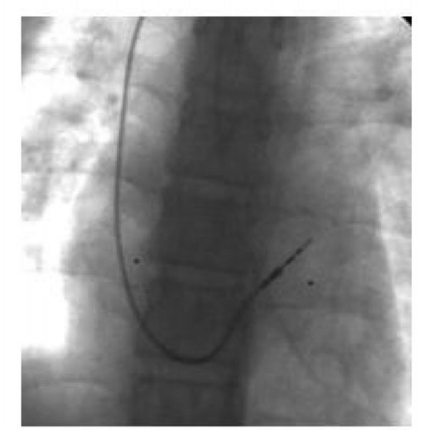

Figure 2. Exemplary fluoroscopic appearances of lead implant sites along the mapped right ventricular septal areas as viewed in the frontal plane. (A) Apex. (B) Low septum. (C) Midseptum. (D) Infundibular/outflow septum.

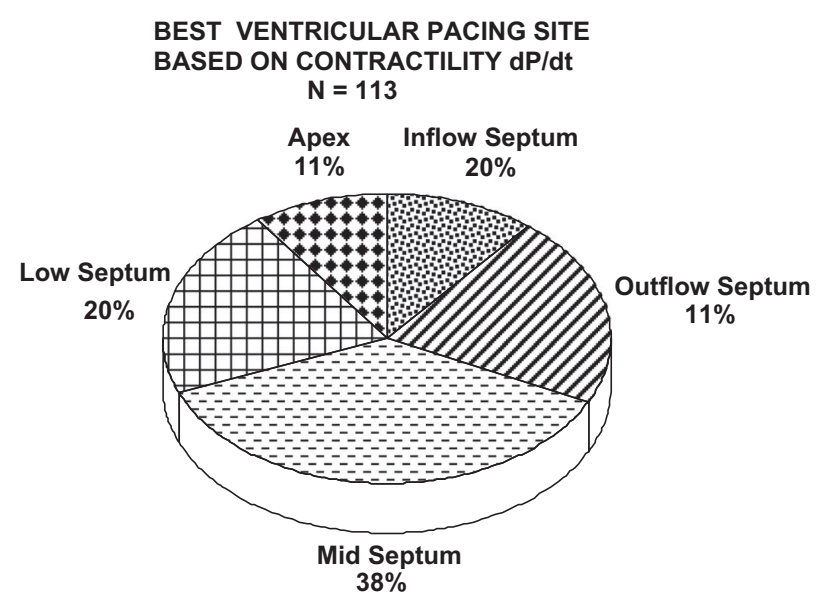

Figure 3. Pie diagram illustrating percent breakdown of the most optimal ventricular implant sites overall, based on measured contractility index $(d P / d t)$ regardless of anatomy.

\section{Results}

A total of 113 consecutive patients, aged 2-51 (mean 17 years) were studied. Of these, 46 had various repaired congenital heart defects that included ventricular septal defects (16 patients) or tetralogy of Fallot (TOF; 10 patients) and Dtransposition of the great arteries with the Mustard intraatrial baffle (nine patients) or Jatene arterial switch (five patients) procedures. Other repaired CHD included valve surgeries (six patients). The nonsurgical patients included congenital AVB (CAVB), of whom three patients had congenitally corrected transposition or L-TGA. A preexisting right ventricular free wall epicardial pacing system was present in 26 patients. There were no adverse events following the temporary pacing protocol, which added approximately 20 minutes to the implant time. Permanent pacemaker implant was not associated with any complications. There was neither excessive bleeding nor pocket hematomas. Depending on design, all preselected implant sites were achieved without complications using appropriately curved stylets or, in instances of the M3830 lead (60\% of leads used), the Select Site ${ }^{\mathrm{TM}}$ catheter delivery system.

With each patient serving as his/her own control, the most optimal lead implant site producing the best ventricular contractility was found to be variable from right ventricular apex to infundibular/outflow septum among all patients and congenital heart defects. This was especially true in patients with repaired congenital 
Table I.

Best Pacing Implant Site Comparisons (Mean Values \pm SEM)

\begin{tabular}{|c|c|c|c|c|c|c|c|c|}
\hline & Total & $\begin{array}{c}\text { RV } \\
\text { Apex }\end{array}$ & $\begin{array}{l}\text { Low } \\
\text { Septum }\end{array}$ & Midseptum & $\begin{array}{l}\text { Inflow } \\
\text { Septum }\end{array}$ & $\begin{array}{l}\text { Outflow } \\
\text { Septum }\end{array}$ & $\begin{array}{c}\text { Left } \\
\text { Ventricle }^{\dagger}\end{array}$ & $\begin{array}{c}\text { RV } \\
\text { Epicardial }^{\ddagger}\end{array}$ \\
\hline No. of patients & 113 & 11 & 20 & 41 & 22 & 10 & 9 & 26 \\
\hline $\mathrm{dP} / \mathrm{dt}^{\S}$ & $\begin{array}{c}988 \\
\pm 24\end{array}$ & $\begin{array}{c}907 \\
\pm 73.8\end{array}$ & $\begin{array}{l}993 \\
\pm 69\end{array}$ & $\begin{array}{l}1,065 \\
\pm 38\end{array}$ & $\begin{array}{c}960 \\
\pm 49\end{array}$ & $\begin{array}{c}925 \\
\pm 102\end{array}$ & $\begin{array}{l}846 \\
\pm 50\end{array}$ & $\begin{array}{l}800^{*} \\
\pm 75\end{array}$ \\
\hline $\mathrm{dP} / \mathrm{dt} / \mathrm{p}$ & $\begin{array}{c}27 \\
\pm 0.6\end{array}$ & $\begin{array}{c}26 \\
\pm 2.1\end{array}$ & $\begin{array}{r}27 \\
\pm 1.8\end{array}$ & $\begin{array}{c}25 \\
\pm 1.0\end{array}$ & $\begin{array}{c}27 \\
\pm 1.2\end{array}$ & $\begin{array}{c}27 \\
\pm 2.2\end{array}$ & $\begin{array}{c}25 \\
\pm 2.1\end{array}$ & $\begin{array}{l}24^{*} \\
\pm 1.9\end{array}$ \\
\hline $\operatorname{Th}(\mathrm{V})^{\mathfrak{n}}$ & $\begin{array}{c}0.6 \\
\pm 0.3\end{array}$ & $\begin{array}{c}0.6 \\
\pm .06\end{array}$ & $\begin{array}{c}0.6 \\
\pm .09\end{array}$ & $\begin{array}{c}0.7 \\
\pm .05\end{array}$ & $\begin{array}{c}0.6 \\
\pm .05\end{array}$ & $\begin{array}{c}0.9^{*} \\
\pm 0.1\end{array}$ & $\begin{array}{c}0.5 \\
\pm .08\end{array}$ & $\begin{array}{c}0.7 \\
\pm 0.06\end{array}$ \\
\hline $\mathrm{R}$ wave (mv) & $\begin{array}{r}11.9 \\
\pm 0.6\end{array}$ & $\begin{array}{l}10.9 \\
\pm 1.0\end{array}$ & $\begin{array}{l}10.7 \\
\pm 1.4\end{array}$ & $\begin{array}{r}13.2 \\
\pm 1.1\end{array}$ & $\begin{array}{l}10.5 \\
\pm 1.2\end{array}$ & $\begin{array}{l}14.9 \\
\pm 2.4\end{array}$ & $\begin{array}{c}9.8 \\
\pm 1.7\end{array}$ & $\begin{array}{c}10.7 \\
\pm 1.2\end{array}$ \\
\hline QRS (ms) & $\begin{array}{c}124 \\
\pm 2.2\end{array}$ & $\begin{array}{c}122 \\
\pm 12.6\end{array}$ & $\begin{array}{r}128 \\
\pm 4.8\end{array}$ & $\begin{aligned} & 124 \\
\pm & 3.0\end{aligned}$ & $\begin{array}{l}113^{*} \\
\pm 3.8\end{array}$ & $\begin{array}{c}138 \\
\pm 7.9\end{array}$ & $\begin{array}{c}128 \\
\pm 8.4\end{array}$ & $\begin{array}{l}146^{*} \\
\pm 6.9\end{array}$ \\
\hline EDP (mm Hg) & $\begin{array}{c}10 \\
\pm 0.3\end{array}$ & $\begin{array}{c}11 \\
\pm 1.4\end{array}$ & $\begin{array}{l}9 \\
\pm 0.8\end{array}$ & $\begin{array}{c}9 \\
\pm 0.5\end{array}$ & $\begin{array}{c}10 \\
\pm 0.7\end{array}$ & $\begin{array}{c}14 \\
\pm 1.7\end{array}$ & $\begin{array}{c}9 \\
\pm 0.8\end{array}$ & $\begin{array}{c}11 \\
\pm 0.9\end{array}$ \\
\hline
\end{tabular}

${ }^{*} \mathrm{P}<0.05$ compared with all other groups.

†Paced venous ventricle with "left" morphology (D/L TGA), dP/dt of systemic "right" ventricle.

$\ddagger$ Prexisting RV epicardial systems.

$\S \mathrm{mm} \mathrm{Hg}$-s; systemic ventricle function.

"At $0.5 \mathrm{~ms}$.

POST-SEPTAL DEFECT REPAIR

$$
\mathbf{N}=\mathbf{2 6}
$$

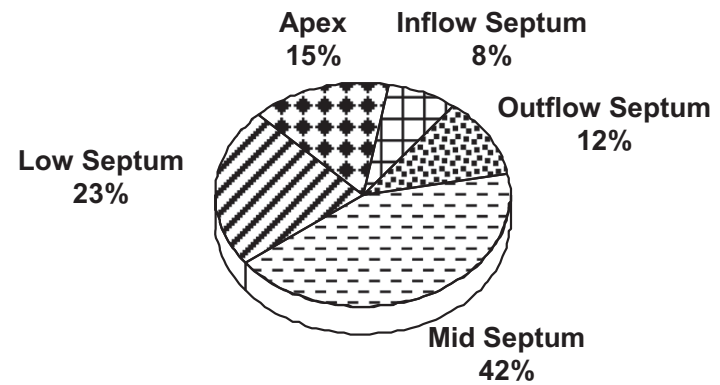
NON-SURGICAL
$\begin{gathered}\text { CONGENITAL HEART } \\ \text { N }=67\end{gathered}$

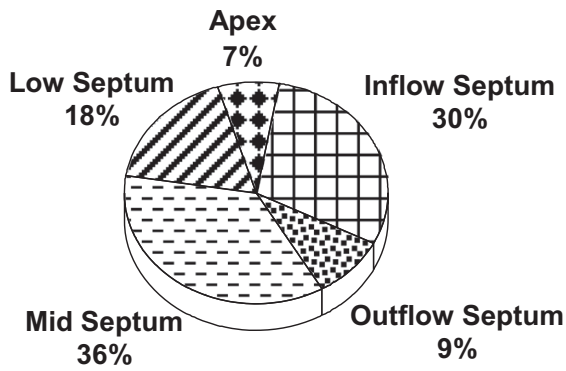

Figure 4. Pie diagrams showing percentage of best right ventricular implant sites based on contractility by implant location among patients specifically with repaired septal defects/surgical AV block and nonsurgical (e.g., congenital AVB) congenital heart. (Left) Patients with septal defect repair, including tetralogy of Fallot. (Right) Patient without previous surgery.

heart disease. As seen in Table I, indices of contractility $(\mathrm{dP} / \mathrm{dt}, \mathrm{dP} / \mathrm{dt} / \mathrm{P})$ did differ between sites among patients with or without CHD. Measured differences in contractility associated with pacing sites ranged from $5 \%$ up to $31 \%$ (mean 12\%) between the best and worst implant site location per patient. When compared by site (apex, low septum, etc.), no intracardiac site was shown to be more or less optimal than another with mean contractility differences of $11-13 \%$ per site, indicating that patient factors and not site, per se, are the most important variables. Analysis of the most optimal pacing site based on anatomy are shown in Figures 1-4. Among all patients, the midseptal locations, approximating either the defined midseptal moderator band, inflow or low septal regions, were associated with the best hemodynamic responses compared with either the ventricular apical and distal infundibulum/outflow sites. Among patients with repaired ventricular septal defects, the inflow septal region was shown to be less optimal with 
only $8 \%$ of patients exhibiting the best contractility measurements from that paced location. This group also showed that in $15 \%$ of patients, the apical site offered improved contractility. Since there were only nine patients with DTGA/Mustard repair, any valid comparison of LV sites was not possible. Following permanent lead insertion, repeat contractility measurements were comparable to the prelead implant value.

End-diastolic pressures were not significantly different among mapped sites or when compared with intrinsic values. Although there were some variations in paced QRS duration, the only significant differences were seen among those patients with inflow septal pacing in which the QRS shortened and those with preexisting right ventricular epicardial pacing in which the QRS was prolonged $(\mathrm{P}<0.05)$. Epicardial right ventricular pacing was also associated with significantly worse contractility compared with all other sites $(\mathrm{P}<0.05)$. Sites between the midseptal and apical regions were typically associated with a leftward or superior QRS axis, whereas regions between the midmoderator and semi-lunar valve exhibited a more normalized QRS axis. Sensed implant R waves did not differ significantly between sites. Implant thresholds also were comparable between sites except for the outflow region that was higher but still acceptable at less than 1 Volt.

Follow-up evaluations from 5 years to 11 years (mean 7) did not demonstrate any lead dislodgements or appreciable changes in pacing threshold, R-wave sensing, or lead impedances regardless of pacing site or lead used. No reprogramming to compensate for any adverse lead performance problems was required. There was no evidence of clinical contractility deterioration among patients. Follow-up echocardiographic measurements, as a whole, did not illustrate any appreciable differences from initial values. During this time interval, follow-up hemodynamic catheterization studies were performed in six patients, 16-22 years of age (mean 18), at the time of generator replacement for battery depletion or other clinical issues. Repeat measurements with temporary pacing showed that the initially chosen implant site still demonstrated the best contractility with site comparisons of $\mathrm{dP} / \mathrm{dt}$ ranging from $5 \%$ to $33 \%$ (mean $22 \%$ ).

\section{Discussion}

As an index of ventricular function, $\mathrm{dP} / \mathrm{dt}$ reflects peak contractility, elasticity, and ventricular dimensions. Since it is attained before aortic valve opening, the measured value is independent of afterload. However, there may be some preload dependence. For this reason, the value of $\mathrm{dP} / \mathrm{dt} / \mathrm{p}$, which incorporates developed isovolumic pressure and negates any preload interference, was also studied with the patient in a sedated physiologic steady state with controlled respirations. In this regard, initiation of ventricular contraction via pacing site was the more important variable that any atrial contribution to ventricular filling, per se. Contractility was measured during single site ventricular pacing. Current physiologic recording catheterization laboratory equipment permits relatively simple and reproducible on-line acute evaluations of preimplant LV contractility performance indices, which may translate into chronic clinical benefits. Although the mapping protocol may lengthen the implant procedure time when compared with a typical ventricular apical lead implant not accompanied by hemodynamic measurements, optimization of lead implant offers the potential for improved chronic ventricular performance.

Initial pacemaker application concerns of lead stability, integrity, pacing threshold, and generator battery/circuitry have been largely satisfied over the past three decades of technologic advances. ${ }^{26}$ Ventricular electrical activation and consequent LV function have only recently enjoyed a resurgence of interest, due primarily to the increasing body of evidence that chronic right ventricular apical pacing can be associated with adverse physiology and altered cardiac histopathology that may contribute to patient morbidity and mortality. These concepts have led to interest in septal, alternate, or select-site as well as multisite pacing to either improve the failing myocardium or prevent future pacing-related dysfunction. ${ }^{27}$ The multiple recent trials of cardiac resynchronization therapy (CRT) or BiV pacing substantiate improvement in ischemic and nonischemic (or idiopathic) myocardial dysfunction in some selected patients with advanced heart failure. ${ }^{28,29}$ These concepts have recently been applied in a very limited capacity to younger patients, with and without congenital heart defects, as a potentially effective therapy to improve hemodynamics or as an alternative to heart transplant. ${ }^{30-32}$ However, $\mathrm{CRT} / \mathrm{BiV}$ pacing is a retroactive approach for an already depressed contractile condition and may not be as necessary in younger patients as in older patients with myocardial dysfunction, if more precise ventricular pacing sites that optimize ventricular performance can be established from the time of initial pacemaker application.

In CRT trials, a 5-10\% increase in LV ejection fraction (EF) has been indicated as a marker for a positive response to therapy. However EF, especially among patients with regional asynchrony, such as with preexisting paced rhythm, bundle branch block, or postsurgical septal patches, becomes less accurate and valid as an effective 
measurement of contractility. ${ }^{33}$ As a result, this study did not utilize any indirectly calculated echocardiographic parameters of measured function, but relied on more direct hemodyanmic measurements. In this regard, contractility indices varied from $5 \%$ to $31 \%$ between implant sites. Such changes are in accord with published CRT reports indicating benefit, and, as such, the site associated with the best contractility response should be sought to optimize paced myocardial function.

Prevention of myocardial dysfunction by a more proactive approach to initial pacemaker application is a less-established clinical application than standard implant protocols. However, such an approach is perhaps more applicable to younger patients in whom pacing may be anticipated to be required for a more protracted period than in elderly patients. In this regard, initial lead implant optimization with direct measurements of physiologic responses appears to be a prudent approach, as there was up to a $31 \%$ difference in measured contractility indices between the best and worst implant site. Although, ideally, all patients would have undergone a follow-up hemodynamic study after implant to confirm site efficacy, due to clinical issues, only a few were able to be studied. Nevertheless, the initially chosen site still exhibited the most optimal contractility response among those patients. This study also confirms previous reports of adverse contractility associated with RV epicardial free wall pacing. ${ }^{34}$

Not unexpectedly, CHD patients with surgically repaired septal defects (including TOF) encompassed the largest group requiring permanent pacing. Unfortunately, other represented CHD anatomies were too few to make any definitive comparisons of optimal pacing site. That will be work for future studies. Of interest, and perhaps not unexpected, the RV apex was shown to be the more optimal implant site in a few patients with repaired CHD, especially considering septal patch materials used for septal defect closure. Following septal defect repair, extensive fibrotic changes along the septum may preclude any effective lead implant as well as contribute to regional wall motion abnormalities. Of these 11 patients with optimal apical pacing sites, nine had previous septal defects repaired resulting in regional septal wall dyssynchrony. This finding does emphasize the need to ascertain site optimization prior to simply assuming, for example, that RV apical pacing is potentially detrimental in all patients. In addition, although the numbers are small, patients in whom the systemic ventricle was of a "right" ventricle morphology, such as among those following the Mustard repair for D-TGA, showed decreased contractility when compared to other CHD with a systemic "left" ventricle. Larger numbers of patients will be required to determine any significance among this group and others with repaired CHD. Nevertheless, since early heart failure can be expected in some adult patients with various repaired congenital heart defects, optimization of paced myocardial function should be sought to potentially improve chronic clinical well-being.

Although many studies have advocated right ventricular outflow tract pacing, precise definition of this anatomic region is often not specified. For this reason, any location from the low septal to subpulmonic infundibular regions can be interpreted as the outflow tract. This study subdivides the septal surface into regions. Results confirm that this is a diverse area and pacing from each site results in different ventricular performance indices as previously reported from pressure-volume loop studies. ${ }^{19}$ Since ventricular anatomy is not a constant variable, especially in CHD, and normal variations in structure are commonly found, for example, absence of an identifiable moderator band, it is mandatory for a more precise definition of the implant site. This especially applies to patients with diverse cardiac anatomy. It was reassuring that the current generation of low threshold, steroid-eluting pacing leads exhibited stable performances regardless of implant site.

Precision of implant location, based on several variables, can provide a better approach to implant. QRS duration may have some association with myocardial performance as previously reported. However, duration alone may not be the best indicator of mechanical contractility. ${ }^{34}$ This was confirmed in this study. Except for the inflow site, QRS duration was not found to be of any appreciable value in correlation with contractility. As a result, other invasive and noninvasive parameters of contractility such as conductance, pressure-volume loops, tissue Doppler, and velocity-time integral have been utilized with variable success. However, use of these measured indices can significantly increase implant time as well as the requirement for equipment not necessarily found in standard cardiac catheterization laboratories. This study utilized readily available on-line $\mathrm{dP} / \mathrm{dt}$ and $\mathrm{dP} / \mathrm{dt} / \mathrm{p}$ measurement values, which are relatively pre- and afterload insensitive, permitting technical ease by use of standard intravascular catheters with objective findings. Unfortunately, to date, there has been no concise agreement on the single best implant variable that has been shown to predict chronic effects of physiologic performance. More prospective studies will be required in this regard. 


\section{Study Limitations}

This is the first study that presents a proactive approach to selective transvenous ventricular lead implantation in the young, with and without repaired CHD, based on contractility response. Although this technique requires a hemodynamic catheterization study, which is recommended for any patient with repaired congenital heart, the use of standard cardiac catheterization hemodynamic measuring equipment permits ease of obtaining contractility indices values. However, originating from a single congenital heart center, patient numbers are limited when compared with other published multicenter studies. In addition, due to patient care issues, follow-up contractility measurements could only be performed when indicated for other reasons, such as generator replacement. As a result, there are limited chronic comparisons. An expanded study in patients from multiple congenital heart centers will be required before any definitive standards for lead implant in the patients with and without structural congenital heart defects can be determined. And since adverse effects of pacing may require many years before any adverse changes are expressed by known objective noninvasive clinical testing, an extended follow-up interval will be required to determine if select site pacing based on initial

\section{References}

1. Furman $S$, Robinson $G$. The use of an intracardiac pacemaker in the correction of heart block. Surgery 1961; 49:98-108.

2. Furman S, Young D. Cardiac pacing in children and adolescents. Pediatr Cardiol 1977; 39:550-558.

3. Gillette P. Recent advances in mechanisms, evaluation, and pacemaker treatment of chronic bradydysrhythmias in children. Am Heart J 1981; 102:920-929.

4. Ennker J, Stegmann T, Luhmer I, Oelert H. Risks and benefits of cardiac pacing in children. Int J Cardiology 1985; 8:125134.

5. Prinzen FW, Augustijn CH, Arts T, Allessie MA, Reneman RS. Redistribution of myocardial fiber strain and blood flow by asynchronous activation. Am J Physiol 1990; 259:330-338.

6. Peschar M, De Swart H, Michels KJ, Reneman RS, Prinzen FW. Left ventricular septal and apex pacing for optimal pump function in canine hearts. J Am Coll Cardiol 2003; 41:1218-1226.

7. Tse HF, Lau CP. Long-term effect of right ventricular pacing on myocardial perfusion and function. J Am Coll Cardiol 1997; 29:225232.

8. Tantengco MVT, Thomas RL, Karpawich PP. Left ventricular dysfunction after long-term right ventricular apical pacing in the young. J Am Coll Cardiol 2001; 37:2093-2100.

9. Adomian GE. Myofibrillar disarray produced in normal hearts by chronic electrical pacing. Am Heart J 1986; 112:79-83.

10. Karpawich PP, Rabah R, Haas JE. Altered cardiac histology following apical right ventricular pacing in patients with congenital atrioventricular block. Pacing Clin Electrophysiol 1999; 22:13721377.

11. Marrus S, Andrews C, Cooper D, Faddis M, Rudy Y. Repolarization changes underlying long-term cardiac memory due to right ventricular pacing: Noninvasive mapping with electropcardiographic imaging. Circ Arrhythm Electrophysol 2012; 5:773781.

12. Yu C, Fung W, Lin H, Zhang Q, Sanderson J, Lau CP. Predictors of left ventricular reverse remodeling after cardiac resynchronization implant variables will prevent or lessen reported adverse changes. The population of adults with $\mathrm{CHD}$ is only increasing. Optimization in all aspects of therapeutic care delivery is mandatory.

\section{Conclusions}

Although not universally applicable to all patients, a midseptal ventricular implant site may offer the best paced ventricular contractility. However, "best-site" is patient and CHD variable. More precise lead implant locations with measurement of physiologic response should be explored to both optimize systemic ventricular performance and potentially prevent eventual adverse changes. Such studies add little extra time to an implant. Optimizing lead implantation based on systemic ventricular physiologic responses, at least acutely, may be a promising approach to lead implant protocols. Chronic follow-up will be required to determine if the reported adverse changes associated with pacing also occur following pacing at these alternative selected sites in a growing patient population with and without congenital heart defects.

Acknowledgment: The author wishes to thank Paul Webster RTR and the technical and nursing catheterization laboratory staff for assistance in data acquisition. therapy for heart failure secondary to idiopathic dilated or ischemic cardiomyopathy. Am J Cardiol 2002; 9:684-688.

13. Wiggers J. The muscle reactions of the mammalian ventricles to artificial surface stimulation. Am J Physiol 1925; 73:346-378.

14. Fung J, Yu C. Should we switch to RVOT pacing for all now? Not yet. J Cardiovasc Electrophysiol 2010; 21:1127-1129.

15. Gong X, Su Y, Pan W, Cui J, Liu S, Shu X. Is right ventricular outflow tract pacing superior to right ventricular apex in patients with normal cardiac function? Clin Cardiol 2009; 32:695699.

16. Janousek J, Van Geldorp I, Krupickova S, Rosenthal E, Nugent K, Tomaske M, Fruh A, et al. Permanent cardiac pacing in children: Choosing the optimal pacing site: A multicenter study. Circulation 2013; 127:613-623.

17. Padeletti L, Pieragnoli P, Ricciardi G, Perrotta L, Grifoni G, Porciani C, Lionetti V, et al. Acute hemodynamic effect of left ventricular endocardial pacing in cardiac resynchronization therapy: Assessment by pressure-volume loops. Circ Arrhythm Electrophysiol 2012; 5:460-467.

18. Mason D, Braunwald E, Covell J, Sonnenblick E, Ross J. Assessment of cardiac contractility: The relation between the rate of pressure rise and ventricular pressure during isovolumic systole. Circulation $1971 ; 44: 47-58$

19. Lieberman R, Padeletti L, Schreuder J, Jackson K, Michelucci A, Colella A, Eastman W, et al. Ventricular pacing lead location alters systemic hemodynamics and left ventricular function in patients with and without reduced ejection fraction. J Am Coll Cardiol 2006; 48:1634-1641.

20. Delnoy P, Ottervanger J, Vos D, Elfan A, Misier A, Beukema W, Steendijk $\mathrm{P}$, et al. Upgrading to biventricular pacing guided by pressure-volume loop analysis during implantation. J Cardiovasc Electrophysiol 2011; 22:677-683.

21. Karpawich PP, Gates J, Stokes K. Septal His-purkinje ventricular pacing in canines: A new endocardial electrode approach. Pacing Clin Electrophysiol 1992; 15:2011-2015. 


\section{KARPAWICH, ET AL.}

22. Deshmukh P, Romanyshyn M. Direct His-bundle pacing: Present and future. Pacing Clin Electrophysiol 2004; 27:862-870.

23. Karpawich PP, Mital S. Comparative left ventricular function following atrial, septal and apical single chamber pacing in the young. Pacing Clin Electrophysiol 1997; 20:1983-1988.

24. Giudici M, Karpawich P. Alternative site pacing: It's time to define the terms. Pacing Clin Electrophysiol 1999; 22:551-553.

25. Yang S, Bentivoglio L, Maranhao V, Goldberg H (eds.). From Cardiac Catheterization Data to Hemodynamic Parameters. Philadelphia, PA, F.A. Davis Company, 1988.

26. Khan A, Zelin K, Karpawich PP. Performance of the lumenless 4.1-Fr diameter pacing lead implanted at alternative pacing sites in congenital heart: A chronic 5-year comparison. Pacing Clin Electrophysiol 2010; 33:1467-1474.

27. Mond HG. The road to right ventricular septal pacing: Techniques and tools. Pacing Clin Electrophysiol 2010; 33:888-898.

28. Kass DA. Ventricular resynchronization: Pathophysiology and identification of responders. Rev Cardiovasc Med 2003; 4(Suppl 2):S3-S13.

29. Abraham WT.Cardiac resynchronization therapy for heart failure: Biventricular pacing and beyond (clinical trials). Curr Opin Cardiol $2002 ; 17: 346-352$.
30. Janousek J, Vojtovic P, Hucin B, Tláskal T, Gebauer RA, Gebauer R, Matejka T, et al. Resynchronization pacing is a useful adjunct to the management of acute heart failure after surgery for congenital heart defects. Am J Cardiol 2001; 88:145152.

31. Dubin A, Janousek J, Rhee E, Strieper M, Cecchin F, Law I, Shannon K. Resynchronization therapy in pediatric and congenital heart disease patients. J Am Coll Cardiol 2005; 46:277283.

32. Vanagt W, Verbeek XA, Delhaas T, Mertens L, Daenen WJ, Prinzen FW. The left ventricular apex is the optimal site for pediatric pacing Correlation with animal experience. Pacing Clin Electrophysiol 2004; 27:837-843.

33. Blyakhman F, Naidich A, Kolchanova S, Sokolov S, Kremleva Y, Chestukhin V. Validity of ejection fraction as a measure of myocardial functional state: Impact of asynchrony. Eur J Echocardiogr 2009; 10:613-618.

34. Gebauer RA, Tomek V, Kubus P, Razek V, Matejka T, Salameh A, Kostelka M, et al. Differential effects of the site of permanent epicardial pacing on left ventricular synchrony and function in the young: Implications for lead placement. Europace 2009; 11:16541659 